\title{
Continuous Analgesia Using a Multi-Holed Catheter in Serratus Plane for Thoracic Surgery
}

\section{TO THE EDITOR}

We read with great interest Mandabushi and colleagues commentary about Serratus Anterior Plane Block as a New Analgesic Technique for Post-Thoracotomy Pain (1). They concluded that SAP block is very easy to perform and provides excellent analgesia in patients undergoing thoracotomy with minimal side effects. We would like to describe its possible use and limitations for analgesic control for thoracic surgery. We have performed a serratus anterior plane (SAP) block using a multi-holed catheter (MHC) for continuous local anesthetic (LA) infusion in 6 patients undergoing videoassisted thoracoscopic surgery (VATS) for lobectomy and our experience, although very limited, allows us to draw interesting conclusions.

Patients undergoing major open postero-lateral thoracotomy can suffer severe pain, which is sometimes hard to control with conventional analgesia that may develop into respiratory complications (2). Despite changes in the surgical technique of VATS with the use of small incisions (minithoracotomy about $5 \mathrm{~cm}$ and an accessory $1 \mathrm{~cm}$ incision), the possibility of rib damage, intercostal nerves injury, pulmonary parenchyma or pleura incision and pleura irritation (caused by haemostatic and sealer surgical glue applied to cover parenchyma defects and pleural drainage tube contact during postoperative period) can still be a problem. Despite several approaches for the pain management for thoracic surgery, the SAP block has as main purpose to cover chest skin incision pain by a sensory blockade allowing multiple perforating intercostal cutaneous branches of the intercostal nerves by LA injection contained between intercostals and serratus muscles interfascial plane (3). Whereas visceral pleura lacks of innervation, parietal sensory innervation is delivered from intercostal (subcostal branches) and phrenic nerves, in theory not attached by SAP block. Even though SAP block is performed in a superficial plane, given the proximity $(0.5-1 \mathrm{~cm})$ where LA is deposited on the pleura, diffusion across intercostal musculature may play an important role reaching extrapleural level and providing parietal pleura analgesia. This can be explained because of the better LA dispersion with MHC with a continuous infusion in the SAP and the evidence

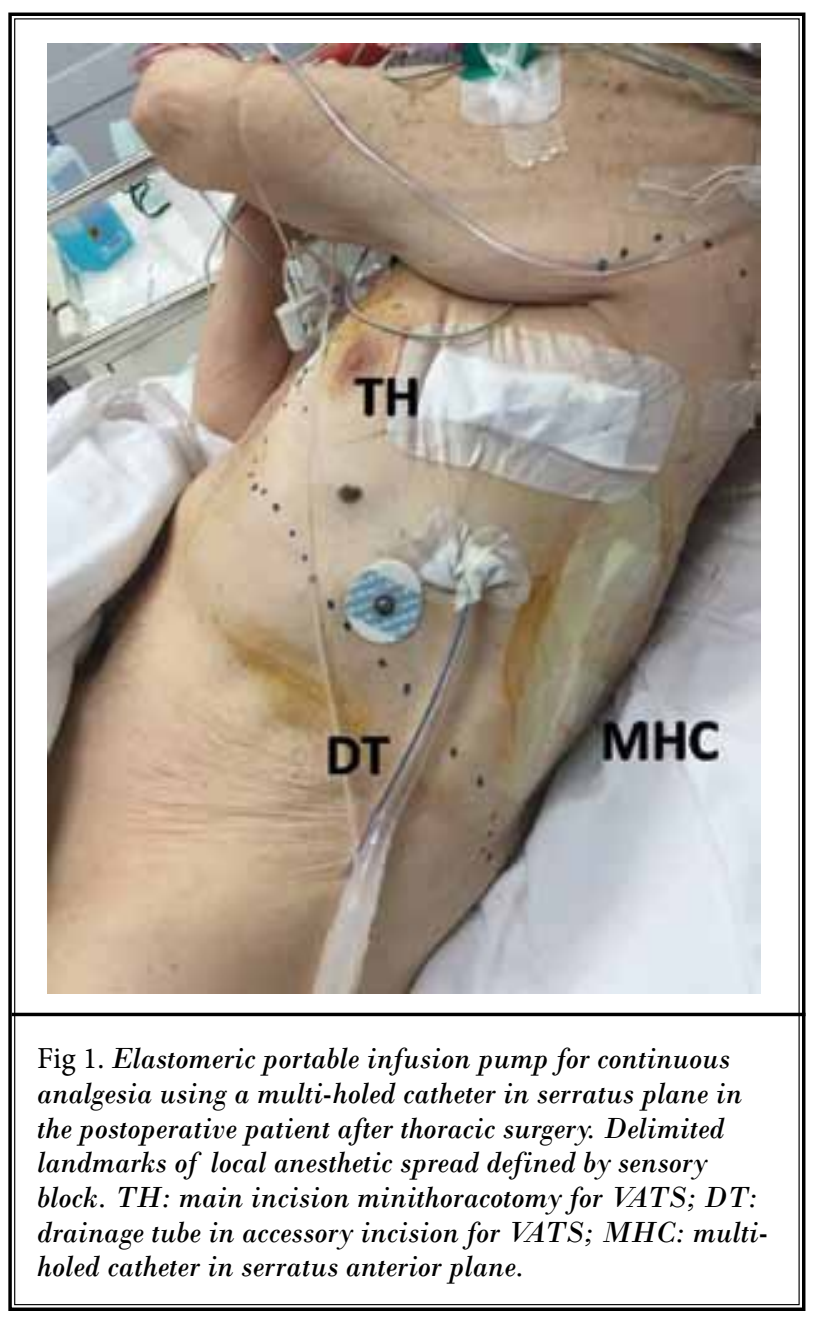

that muscles and fascias are porous tissues. To verify the existence of this effect, we conducted a series of cases with methylene blue instillation during SAP block performance for VATS to image the tissue coloration from an intrathoracic vision. We concluded that a potential spread exists through intercostals musculature to extrapleural space so LA reaching might be volume dependent. Another questionable matter would be the amount of LA used for a successful SAP block (4). Local anesthetic systemic toxicity may be a concern because of large doses of drug managed and due to the prox- 
imity to the intercostal space (high-absorption area). Intercostal cutaneous branches are arranged into an interfascial muscular plane that determines a volume dependent efficacy block, but it is important to note that sensitive nerve do not require the same amount of anesthetic to afford the blockade because of the fiber's nerve size. Despite being a small and poorly distensible plane, MCHs placed between the musculature [usually used in surgical wounds infiltration (5)] allows us to achieve greater blockage areas compared to classical epidural catheters. MCHs have side holes and a blind tip, increasing LA dispersion over interfascial plane to attain an optimal regional sensory blockade with LA continuous infusions without requiring additional bolus. Further, respiratory movements permit LA to be extensively dispersed within this plane contributing to the block success (Fig 1). In addition, the midaxillary approach blocks lateral cutaneous branches of the intercostal nerves, but dorsal and ventral diffusion could also provide posterior and anterior cutaneous branches block. To summarize, this asseverations allowed us to reduce the final LA administered and enabled the use of lower concentrations, avoiding toxec doses, but more studies are needed to evaluate plasma levels of local anesthetic following a SAP block to determine the real LA absorption.

We believe following our experience that SAP block may be a suitable alternative to thoracic epidural analgesia or even the first option because it may theoretically be associated with a less desirable side-effect profile mainly in those cases where we want to avoid neuroaxial blocks. Although LA injection is carried out in a superficial plane (sensory block), we suggest that the benefit of the SAP block for VATS lobecotomy is due to the LA diffusion to the extrapleural space using a continuous infusion with a MHC. This finding suggests the possibility of achieving multiple intercostal nerve blockades with an interfascial and superficial plane block. Further work with this technique is required to determine the precise LA volume and timing of the continuous infusion. To conclude, we agree Rajashree and colleagues with conclusions about SAP effectiveness for thoracotomy pain but we successfully used an alternative approach with a $\mathrm{MCH}$ for continuous analgesia in SAP proving that it can offer more advantages due to LA spread to cover both skin incisions and to reach pleural analgesia.

\author{
Marta Carrió Font, MD \\ Hospital General Universitario de Alicante \\ Pintor Baeza, 11 \\ 03010 Alicante, Spain \\ E-mail: martetacarrio@gmail.com \\ Jose Navarro-Martinez, MD \\ Hospital General Universitario de Alicante \\ Pintor Baeza, 11 \\ 03010 Alicante, SPAIN
}

Sergio Bolufer Nadal, MD

Hospital General Universitario de Alicante

Pintor Baeza, 11

03010 Alicante, Spain

Carlos Gálvez Muñoz, MD

Hospital General Universitario de Alicante

Pintor Baeza, 11

03010 Alicante, Spain

María Galiana-Ivars, MD

Hospital General Universitario de Alicante

Pintor Baeza, 11

03010 Alicante, Spain

Pablo Cuesta Montero, MD

Hospital General Universitario de Alicante

Pintor Baeza, 11

03010 Alicante, Spain

\section{References}

1. Madabushi R, Tewari S, Gautam S, Agarwal A. Serratus anterior plane block: A new analgesic technique for post-thoracotomy pain. Pain Physician 2015; 18: 4214

2. Weissman C. Pulmonary function after cardiac and thoracic surgery. Anesth Analg 1999; 88: 1272-9
3. Broseta A, Errando C, De Andrés J, DíazCambronero O. Serratus plane block: The regional analgesia technique for thoracoscopy? Anaesthesia 2015; 70: 1320-33

4. Barr K. Serratus plane block - is a high volume of local anaesthetic needed? Anaesthesia 2014; 69: 180-94
5. Liu SS, Richman JM, Thirlby RC, Wu CL. Efficacy of continuous wound catheters delivering local aenesthetic for postoperative analgesia: A quantitative and qualitative review of randomized controlled trials. Am Coll Surg 2006; 203: 914-32 


\section{In Response to Font et al}

We read with interest the excellent work done by the authors. It is interesting to note that they used multi-holed catheters for infusion of LA for SAP block. Infusion of drug through multi-holed catheters is known to have a better spread due to their dispersion in more than one direction or plane and thus offer better analgesia (1). However, as the authors have rightly pointed out here, it may be worthwhile to know the safe upper limit for volume and dose of LA required in order to avoid toxic side effects, considering that the intercostal area is a site of high absorption.

Although the authors have stated the observation of dispersion of methylene blue dye across the muscles as seen on thoracoscopy, a figure indicating the same would have been more informative. The use of multiholed catheters seems to offer a wider area of analgesia of the hemithorax, as indicated in the figure. The SAP block is a safer alternative in patients where neuraxial blockade is difficult or is contraindicated. Further studies are required to observe the various patterns of analgesia that can be offered with different doses and planes of drug infusion.

Rajashree Madabushi, MD

Department of Anaesthesiology

Sanjay Gandhi Postgraduate

Institute of Medical Sciences,

Rae Bareilly Road, Lucknow 226014

Saipriya Tewari, MD

Department of Anaesthesiology

Sanjay Gandhi Postgraduate

Institute of Medical Sciences,

Rae Bareilly Road, Lucknow 226014

Amita Agarwal, MD

General Hospital

Sanjay Gandhi Postgraduate

Institute of Medical Sciences

Lucknow
Anil Agarwal, MD

Department of Anaesthesiology

Sanjay Gandhi Postgraduate

Institute of Medical Sciences,

Rae Bareilly Road, Lucknow 226014

Sujeet KS Gautam, MD

Assistant Professor

Department of Anaesthesiology,

Sanjay Gandhi Postgraduate

Institute of Medical Sciences

Lucknow - 226014

E-mail: docsksg@gmail.com

\section{References}

1. Stela M, Ljilja S, Ljiljana P, Miroslav B, Kata S. Quality of analgesia with multiversus two few-hole catheters in patients after colorectal surgery. Period Biol; 2011; 113:213 - 216. 